\title{
Giant aneurysm of an aortopulmonary collateral
}

\author{
Vandana Marwah, Tanuj Gupta, Arjun Kalyanpur \\ Teleradiology Solutions, Plot No. 7G, Opp. Graphite India, Whitefield, Bangalore - 560 048; Narayana Hrudayalaya, Bommasandra \\ Industrial Area, Bangalore, India
}

Correspondence: Dr. Vandana Verma Marwah, Flat 3/4D, Alps Estate, Sarjapur Road, Bangalore, India. E-mail: vandyvm@gmail.com

\section{Introduction}

We present an unusual case of a giant aneurysm of an aortopulmonary collateral, in a patient of Tetralogy of Fallot with pulmonary atresia on long-term conservative treatment. Aneurysm of aortopulmonary collateral is an extremely rare finding. Only a few similar cases have been reported in literature.

\section{Case Report}

A 21 -year-old man, known to have Tetralogy of Fallot (TOF), with pulmonary atresia, presented to the pediatric cardiology department with fever for one week. His diagnosis had been established in childhood, but the patient had repeatedly refused surgical treatment. The patient was referred from a peripheral hospital for suspected pleural effusion. He had a history of a brain abscess six years ago, which was removed by trephine aspiration. Clinical examination revealed a loud S1 with a pansystolic murmur in the mitral area. The breath sounds were reduced in the right lower zone with a dull note on percussion.

Echocardiography demonstrated features of TOF and the central pulmonary arteries were not visualized. Pulmonary arterial hypertension was noted. Large aortopulmonary collaterals were seen. Moderate aortic regurgitation was also noted. Biventricular function was good.

A cardiac CT was ordered specifically to assess the central pulmonary arteries, to delineate the aortopulmonary collaterals and to evaluate for any lung pathology, keeping in mind the reduced breath sounds in the right lower zone.

A CT chest with a cardiac protocol was performed. The standard features of TOF were identified. Absence of central pulmonary arteries was confirmed. Also seen were multiple, large aortopulmonary collaterals coursing to both hila and supplying the lungs. A large $(9.2 \mathrm{~cm} \times 5.2$ $\mathrm{cm} \times 8.2 \mathrm{~cm}$ ) saccular aneurysm was seen arising from an aortopulmonary collateral, which was seen coursing initially to the right and then taking a hair pin turn to the left, finally supplying the left lung [Figures 1-4]. The aneurysm was seen extending into the right thoracic cavity up to the right lateral chest wall with resultant atelectasis of the surrounding lung. Compression of the superior vena cava and bronchus intermedius was also seen.

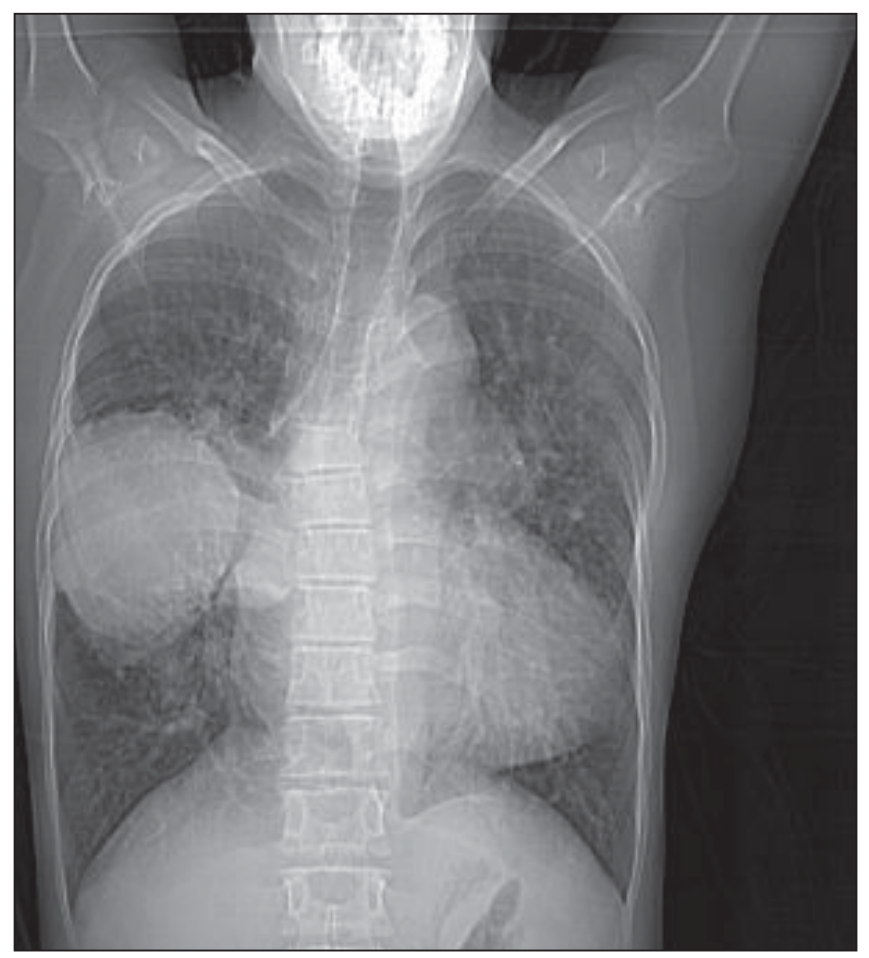

Figure 1: Scanogram demonstrates a large round opacity in the right mid lung, which could represent a mass 

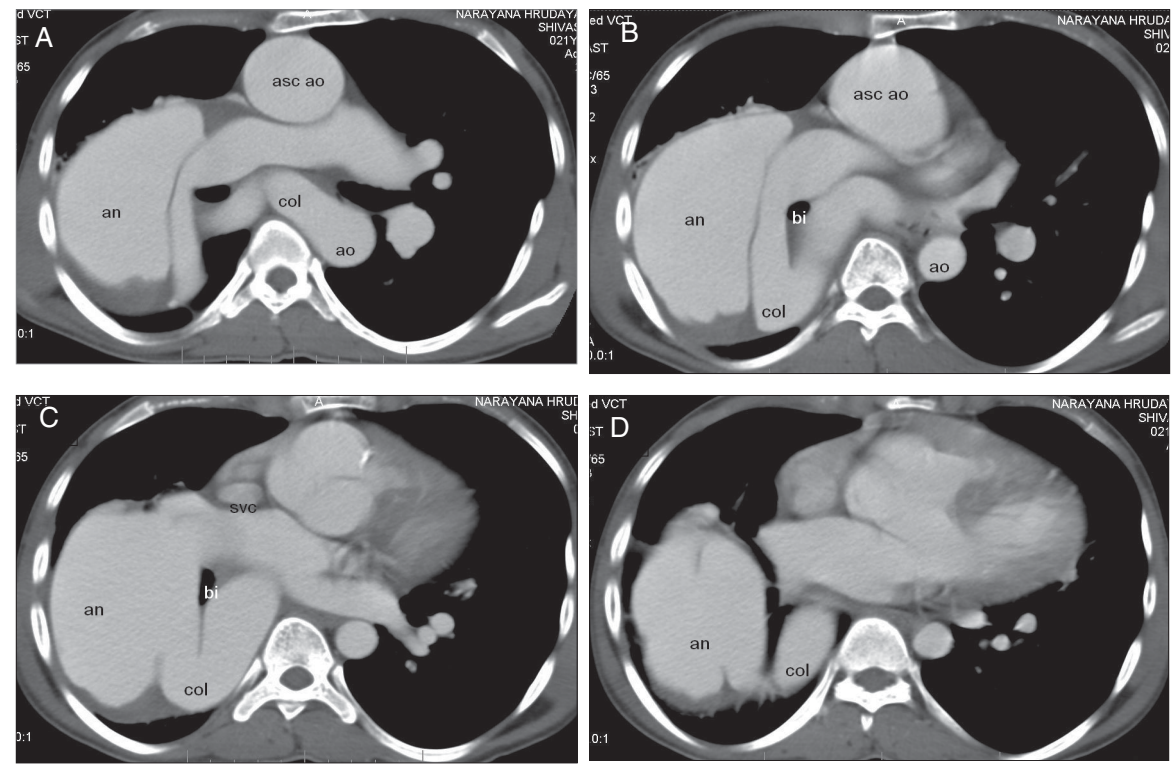

Figure 2 (A-D): The consecutive axial images demonstrate a large collateral arising from the right lateral aspect of the aorta, coursing anterior to the vertebral body and then turning to the left and coursing to the left hilum. The saccular aneurysm is seen as a large, contiguous, contrast opacified structure in the right hemithorax, arising from the collateral, extending laterally to the chest wall with compression of the bronchus intermedius and superior vena cava. an - aneurysm, ao - aorta, bi - bronchus intermedius, col - collateral, svc - superior vena cava, - asc ao - ascending aorta

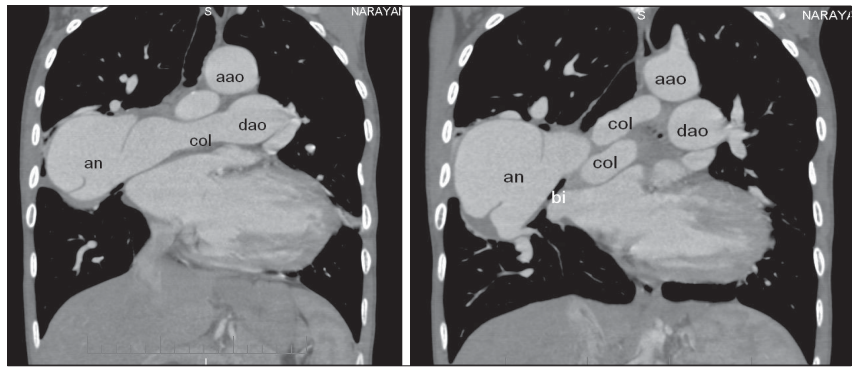

Figure 3: Coronal reformats demonstrating the collateral and the saccular aneurysm. aao - ascending aorta, dao - descending aorta, bi - bronchus intermedius, an - aneurysm, col - collection

\section{Discussion}

Aneurysm of an aortopulmonary collateral is an extremely rare finding. Only a few similar cases have been reported in literature. ${ }^{[1-3]}$ It is seen mostly in untreated cases of TOF with pulmonary atresia and subsequent development of Eisenmenger's syndrome. The pathophysiology is possibly due to the persistent high-pressure state in the collateral circulation. The treatment of choice is urgent occlusion of the aneurysm to prevent severe hemoptysis / catastrophic bleed.

\section{References}

1. Azevedo CF, Hadlich MS, Albuquerque DC, Moll JN. Images in cardiovascular medicine. Untreated tetralogy of Fallot with pulmonary atresia: Giant aneurysm of an aortopulmonary collateral

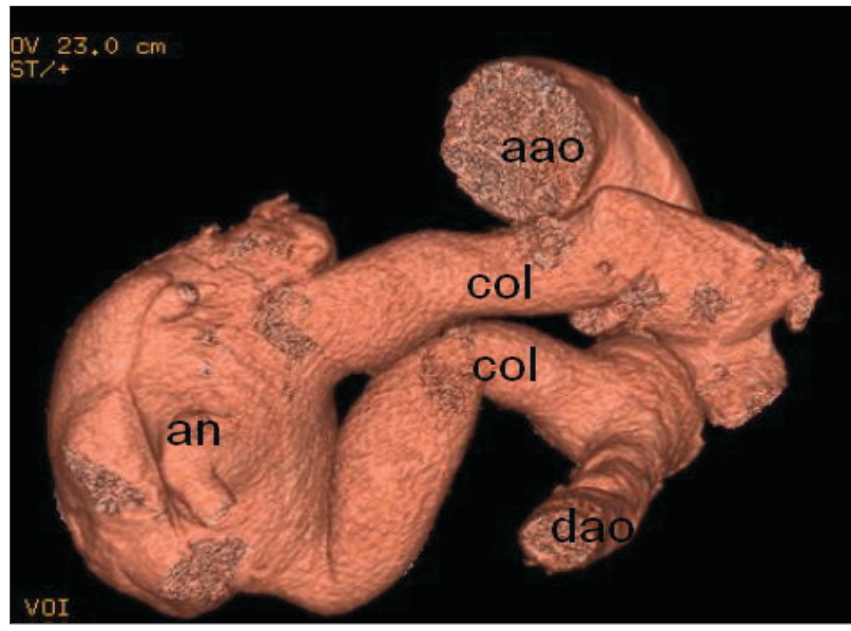

Figure 4: Volume rendered image demonstrating the collateral and the saccular aneurysm. an - aneurysm, aao - ascending aorta, dao - descending aorta, col - collateral

artery demonstrated by magnetic resonance imaging. Circulation 2006;113:e293-4.

2. Radford D, Slaughter R. Coil embolization of iatrogenic arteriovenous fistula from pulmonary collateral artery to subclavian vein. Cardiol Young 2001;11:663-5.

3. Hachiro Y, Sugimoto S, Takagi N, Abe T. Aneurysmal change in an internal mammary artery-pulmonary artery fistula. Ann Thorac Surg 2001;72:1377-8.

Source of Support: Nil, Conflict of Interest: None declared. 